\title{
Anti-VEGF treatment and peripheral retinal nonperfusion in patients with central retinal vein occlusion
}

This article was published in the following Dove Press journal:

Clinical Ophthalmology

I5 February 2017

Number of times this article has been viewed

\author{
Kaveh Abri Aghdam ${ }^{1, *}$ \\ Lukas Reznicek ${ }^{2, *}$ \\ Mostafa Soltan Sanjari' \\ Annemarie Klingenstein ${ }^{2}$ \\ Marcus Kernt ${ }^{2}$ \\ Florian Seidensticker ${ }^{2}$ \\ 'Department of Ophthalmology, \\ Eye Research Center, Rassoul Akram \\ Hospital, Iran University of Medical \\ Sciences, Tehran, Iran; ${ }^{2}$ Department of \\ Ophthalmology, Ludwig-Maximilians \\ University, Munich, Germany \\ *These authors contributed equally \\ to this work
}

Purpose: To evaluate the association between the size of peripheral retinal nonperfusion and the number of intravitreal ranibizumab injections in patients with treatment-naïve central retinal vein occlusion (CRVO).

Methods: Fifty-four patients with treatment-naïve CRVO and macular edema were included. Each patient underwent a full ophthalmologic examination including optical coherence tomography imaging and ultrawide-field fluorescein angiography. Monthly intravitreal ranibizumab injections were applied according to the recommendations of the German Ophthalmologic Society. Two ophthalmologists quantified the areas of peripheral retinal nonperfusion (group $1=$ less than five disc areas, group $2=$ more than five disc areas). Correlation analyses between the size of nonperfusion with best-corrected visual acuity, central subfield thickness, and the number of intravitreal injections were performed.

Results: Best-corrected visual acuity improved significantly after intravitreal injections $(P<0.001$, both groups). Final central subfield thickness after treatment did not significantly differ between both groups ( $P=0.92, P=0.96$, respectively). Mean number of injections in group 1 and group 2 was $4.12 \pm 2.73$ and $9.32 \pm 3.84$, respectively $(P<0.001)$. There was a significant positive correlation between areas of nonperfusion and the number of injections in each group. ( $R=0.97, P<0.001 ; R=0.94, P<0.001$, respectively).

Conclusion: Peripheral retinal nonperfusion in patients with CRVO correlates significantly with the number of needed intravitreal ranibizumab injections. Ultrawide-field fluorescein angiography is a useful tool for detection of peripheral retinal ischemia, which may have direct implications in the diagnosis, follow-up, and treatment of these patients.

Keywords: angiography, central retinal vein occlusion, non-perfusion, retina, wide-field

\section{Introduction}

Central retinal vein occlusion (CRVO) is a common sight-threatening vascular disease with prevalence rates ranging from $0.1 \%{ }^{1}$ to $0.5 \%{ }^{2}$ in the older adult population. CRVO is characterized by occlusion of the central retinal vein and consecutive damming of the venous blood flow. The occlusion may occur at the level of or posterior to the lamina cribrosa. ${ }^{3}$ Despite its prevalence, the pathogenesis of CRVO is not completely understood. A combination of vascular, anatomic, and inflammatory factors may contribute to its pathophysiology. ${ }^{4}$ Current treatment options include intravitreal injections of steroids $^{5}$ or anti-vascular endothelial growth factor (anti-VEGF) agents. ${ }^{6-8}$

Fluorescein angiographic (FA) assessment is the most important tool for an adequate evaluation of the disease severity and for proper classification between the two types of CRVO, ischemic and nonischemic. Ischemic CRVO is associated with a

\footnotetext{
Correspondence: Kaveh Abri Aghdam Department of Ophthalmology, Eye Research Center, Rassoul Akram Hospital, Sattarkhan-Niayesh Street, Tehran I4456-3 I I3I, Iran

Tel +98 21 66558811

Fax +98 21 665I 6999

Email kaveh.abri@gmail.com
} (c) 1 (1) @ 2017 Abri Aghdam et al. This work is published and licensed by Dove Medical Press Limited. The full terms of this license are available at https://www.dovepress.com/terms.php hereby accept the Terms. Non-commercial uses of the work are permitted without any further permission from Dove Medical Press Limited, provided the work is properly attributed. For permission for commercial use of this work, please see paragraphs 4.2 and 5 of our Terms (https://www.dovepress.com/terms.php). 
significant upregulation of VEGF, a poor prognosis for visual acuity, and has a higher incidence of secondary neovascular glaucoma (NVG) ranging from $20 \%$ to $60 \%$ compared to nonischemic CRVO with no risk of developing NVG. ${ }^{9}$ Given these significant differences with various outcomes and risk profiles, it is crucial to differentiate between ischemic and nonischemic CRVO.

Conventional FA images the retinal circulation within the posterior pole. However, it is not known if conventional FA devices are able to capture areas of interest regarding the intraocular metabolism of VEGF, such as the peripheral retina. Earlier findings suggested an association between peripheral retinal ischemia and an increased production of VEGF. ${ }^{10,11}$ Currently, two ultrawide-field systems are commercially available to perform FA and image the peripheral retina. One method uses a wide-field contact lens system (Ocular Staurenghi 230 SLO Retina Lens; Ocular Instruments, Inc, Bellevue, WA, USA) with the Heidelberg retina angiograph (HRA; Heidelberg Engineering $\mathrm{GmbH}$, Dossenheim, Germany). ${ }^{12,13}$ The second available system uses the Optos scanning laser ophthalmoscope (Optos PLC, Dunfermline, UK), which is a special non-contact-lensbased system that provides visualization up to the peripheral retina. ${ }^{14-16}$ It has been shown that ultrawide-field FA (UWFA) is useful for the assessment of several retinal pathologies ${ }^{17}$ including retinal vein occlusions, ${ }^{18}$ diabetes, ${ }^{19,20}$ uveitis, ${ }^{21}$ retinal vasculitis, ${ }^{22}$ choroidal masses,${ }^{23,24}$ retinal detachment, ${ }^{25}$ and retinopathy of prematurity. ${ }^{26}$

The aim of this study was to evaluate a possible association between peripheral retinal nonperfusion obtained with UWFA and the number of intravitreal ranibizumab injections needed in patients with CRVO.

\section{Methods}

\section{Patient selection}

This prospective interventional study was performed between June 1, 2012, and February 1, 2014. The institutional Review Board of Ludwig-Maximilians University in Munich approved the study design, and patients' care adhered to the tenets of the World Medical Association Declaration of Helsinki. All patients gave written informed consent for both participation in the study and for FA. Inclusion criteria were diagnosis of CRVO (as revealed by retinal hemorrhages and dilated retinal veins in all four quadrants of the fundus) with active center-involving macular edema and central subfield thickness $>250 \mu \mathrm{m}$ detected by spectral-domain optical coherence tomography (SD-OCT, Heidelberg Engineering, Heidelberg, Germany).
Patients without macular edema, with previous focal or panretinal photocoagulation, and with degenerative disorders of the posterior pole and/or retinal periphery were excluded. Additional intravitreal injections were administered in the presence of active center-involving macular edema and central subfield thickness $>250 \mu \mathrm{m}$, as determined by SD-OCT. All patients underwent comprehensive ophthalmologic examination that included best-corrected visual acuity (BCVA) measurement, slit-lamp biomicroscopy, applanation tonometry, indirect ophthalmoscopy, and SD-OCT before injections and at each follow-up visit. UWFA using the Optos 200Tx imaging system was obtained in each case before the first injection. All included patients received three intravitreal injections of $0.50 \mathrm{mg}$ ranibizumab (Lucentis ${ }^{\mathrm{TM}}$, Genentech, Inc., South San Francisco, CA, USA and Novartis Pharma AG, Basel, Switzerland) every four weeks and then were examined monthly with follow-up OCT scans of the macula. They received additional intravitreal injections if macular edema was detected by SD-OCT scans at follow-up visits.

\section{Image acquisition}

SD-OCT volume scans $\left(20^{\circ} \times 15^{\circ}\right.$ with 19 horizontal sections, ART 9, SD-OCT, Heidelberg Engineering, Heidelberg, Germany) of the macula were obtained for each study eye to measure the central subfield thickness (CST) in $\mu \mathrm{m}$ by using Heidelberg SD-OCT software; significant macular ischemia was ruled out by UWFA. FA images were acquired approximately one minute (arteriovenous phase) and 4-5 minutes (late venous phase) after intravenous injection using the Optos 200Tx scanning laser ophthalmoscope (Optos PLC) after standard intravenous infusion of $5 \mathrm{~mL}$ of sodium fluorescein $(10 \%)$ by one experienced technician for all included cases.

\section{Image processing and analysis}

FA images were compressed into high-quality jpeg files (Figures 1 and 2) and analyzed for retinal nonperfusion by two experienced ophthalmologists (KAA and FS). The far peripheral retina was defined as the area between the ampullae of the vortex veins and the ora serrata. The widefield color images and wide-field FA images were used to identify the vortex vein ampullae, which were located near the ocular equator. ${ }^{27}$ Based on another published work, we defined retinal nonperfusion as at least five disc areas of hypofluorescence ${ }^{28}$ (representing retinal nonperfusion or capillary dropout) or areas of microvascular pathology (multiple microaneurysms and significant perivascular leakage) on 


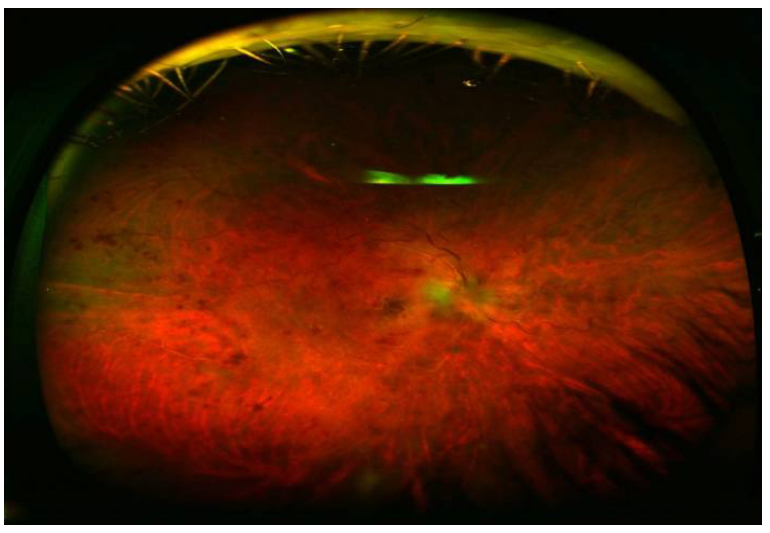

Figure I En face wide-field fundus photograph of the right eye of a patient with CRVO.

Abbreviation: $C R V O$, central retinal vein occlusion.

UWFA. Five disc areas was also the mean size of peripheral retinal nonperfusion of all our patients with CRVO. Because of the effect of upper and lower eyelid and eyelashes, the far periphery in the horizontal plane alone (the nasal and temporal retina) was analyzed.

\section{Data collection}

Collected parameters included demographic information, previous ocular history, number and dates of intravitreal injections, central subfield thickness, peripheral retinal ischemia, central intraretinal fluid, visual acuity, and intraocular pressure (throughout the study period) as well as the occurrence of any complications.

\section{Statistical analysis}

Data were collected and analyzed using SPSS software (version 20.0, IBM Corporation, Armonk, NY, USA). Each graded variable was tested for normal distribution. Nonparametric tests were used to analyze nonnormally distributed data. Paired-sample Wilcoxon signed rank test was used for within group analysis. Mann-Whitney $U$-test was used for intergroup comparison. Spearman rho test was used for correlation analysis. A $P$-value of $<0.05$ was considered statistically significant. Mean \pm standard deviation were used to report the results.

\section{Results}

Fifty-four eyes of fifty-four consecutive patients with treatment-naïve CRVO were enrolled in this study. The average patient age was $74 \pm 11.81$ years (range, $24-86$ years). Twenty-eight patients (52\%) were male, and twenty-three eyes $(42.6 \%)$ were located on the right side. Seven patients $(12.9 \%)$ had known glaucoma, 36 patients $(66.6 \%)$ had systemic hypertension, and 22 patients $(40.7 \%)$ were pseudophakic. Mean follow-up time was $16 \pm 3$ months. All patients had center-involving macular edema confirmed by macular leakage seen in FA and retinal thickening in SD-OCT images. Tables 1 and 2 show the baseline and final BCVA and CST. As shown in the tables, there were significant improvements in the visual acuity within and between groups, whereas changes in CST were not significant. The mean number of intravitreal injections for each patient was $6.64 \pm 3.84$. Twenty-four eyes (44\%) showing less than five disc areas of retinal nonperfusion (group 1) that received a mean number of $4.12 \pm 2.73$ intravitreal ranibizumab injections. Thirty eyes (56\%) showed more than five disc areas (group 2) of retinal nonperfusion in UWFA and received a mean number of $9.32 \pm 3.84$ intravitreal ranibizumab injections $(P<0.001$, Mann-Whitney $U$-test $)$. There was a significant positive correlation between the size of peripheral retinal nonperfusion measured in terms of disc areas and the number of injections in each group.

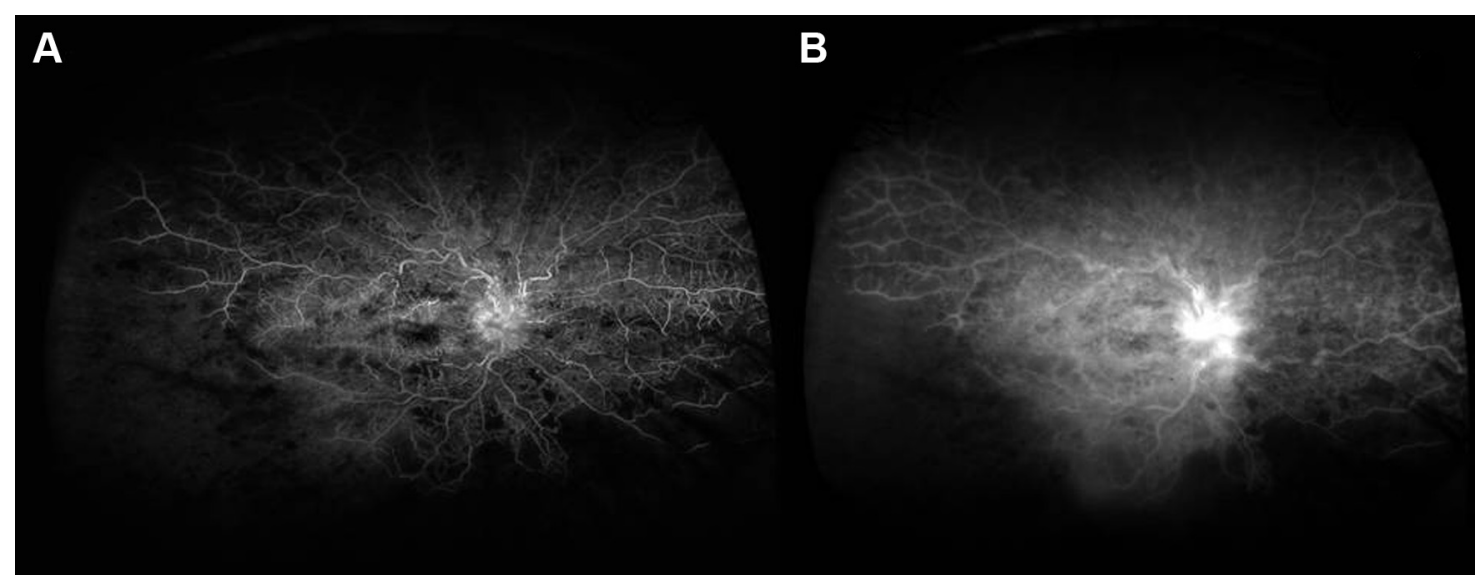

Figure 2 Optos ultrawide-field fluorescein angiogram of the same patient at arteriovenous $(\mathbf{A})$ and late (B) phases.

Note: Fluorescein leakage from the optic nerve head and extensive areas of nonperfusion at temporal and inferotemporal fundus are evident. 
Table I Changes in visual acuity within and between groups

\begin{tabular}{lllll}
\hline $\begin{array}{l}\text { Fields (= disc areas) of } \\
\text { peripheral nonperfusion }\end{array}$ & Number of eyes & Baseline ETDRS BCVA & Final ETDRS BCVA & $\boldsymbol{P}^{\mathrm{a}}$ \\
\hline $0-5$ & 24 & $49.35 \pm 15.98$ & $25.62 \pm 6.02$ & $<0.001$ \\
$>5$ & 30 & $73.26 \pm 12.4$ & $34.38 \pm 8.73$ & $<0.001$ \\
\hline
\end{tabular}

Notes: ${ }^{a}$ Wilcoxon signed-rank test, ${ }^{b}$ Mann-Whitney U-test.

Abbreviations: BCVA, Best Corrected Visual Acuity; ETDRS, Early Treatment of Diabetic Retinopathy Study; P, P-value.

( $R=0.97, P<0.001, R=0.94, P<0.001$, respectively). There was also a significant correlation between the size of retinal nonperfusion and final BCVA in each group. ( $R=0.85$, $P=0.01, R=0.84, P<0.001$, respectively). There was no significant correlation between the size of retinal nonperfusion and final CST in each group. $(R=0.2, P=0.78, R=0.1, P=0.5$, respectively). We did not observe any eye with neovascularization of the disc or neovascularization elsewhere during the observation period; therefore, panretinal photocoagulation was not performed during this study. Furthermore, no cases of injection-related adverse events like retinal detachment or endophthalmitis were encountered.

\section{Discussion}

Venous occlusive disease of the retina is the second most common retinal vascular disorder after diabetic retinopathy. ${ }^{29}$ It typically affects patients between 40 and 80 years of age. ${ }^{30}$ Usually, a decrease of visual acuity is the result of macular edema, which can lead to permanent visual loss and even legal blindness in severe cases.

Applying UWFA, we found a significant correlation between the total number of intravitreal ranibizumab injections and the size of nonperfusion in the peripheral retina: CRVO patients with peripheral retinal nonperfusion received more intravitreal injections for treatment of their macular edema than patients without peripheral retinal nonperfusion. We hypothesized that nonperfused peripheral retina could be a source of increased intraocular VEGF levels with consecutive macular edema. This observation could explain the requirement for more frequent intravitreal antiVEGF injections of in CRVO patients with macular edema and peripheral retinal nonperfusion. This finding is important because an evaluation of the peripheral retina using UWFA may be a prognostic factor and allows the ophthalmologists to estimate the requirement for more or fewer intravitreal injections based on the peripheral retinal perfusion status. Looking at the perfusion of the peripheral retina in those patients, a more precise evaluation of the estimated treatment costs within a defined health care system may be feasible, including a better evaluation of injection-related complications such as endophthalmitis or other complications.

Interestingly, the lack of observed neovascularizations in any of the patients even with extended areas of peripheral retinal nonperfusion might be attributed to good patient compliance, strict follow-up visits every 4 weeks, and immediate treatment in case of detected macular edema.

The results of our study differ from those published by Spaide $^{31}$ and Singer et al. ${ }^{32}$ Spaide evaluated 22 patients treated with ranibizumab for CRVO and found that the area of peripheral nonperfusion was not correlated with the number of injections. The follow-up time of his study was longer than ours, but the sample size was smaller. Singer et a ${ }^{32}$ evaluated 32 patients with retinal vein occlusion and refractory macular edema using SD-OCT and UWFA. Those patients were retreated with intravitreal injections with anti-VEGF and with dexamethasone intravitreal implant. There was no significant difference in the number of anti-VEGF (1.8 vs $1.6, P=0.438$ ) or dexamethasone implant (1.4 vs $1.6, P=0.364$ ) treatments given between the $>10 \%$ and $\leq 10 \%$ nonperfusion groups, nor was there any difference regarding the time to recurrence (3.4 vs 4.3 months, $P=0.440$ ). In Singer et al's series, there were 13 CRVO patients with previously failed contiguous therapy with intravitreal ranibizumab and dexamethasone implant. In contrast, we enrolled previously untreated

Table 2 Changes in central subfield thickness within and between groups

\begin{tabular}{llllr}
\hline $\begin{array}{l}\text { Fields (= disc areas) of } \\
\text { peripheral nonperfusion }\end{array}$ & Number of eyes & $\begin{array}{l}\text { Baseline } \\
\text { CST }(\mu \mathrm{m})\end{array}$ & $\begin{array}{l}\text { Final CST } \\
(\mu \mathrm{m})\end{array}$ & $P^{\mathrm{a}}$ \\
\hline $0-5$ & 24 & $484 \pm 212$ & $344 \pm 160$ & 0.92 \\
$>5$ & 30 & $524 \pm 340$ & $387 \pm 217$ & 0.66 \\
\hline
\end{tabular}

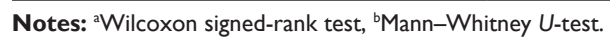

Abbreviations: BCVA, Best Corrected Visual Acuity; CST, central subfield thickness; ETDRS, Early Treatment of Diabetic Retinopathy Study; $P, P$-value. 
(treatment-naïve) patients; furthermore, the number of our cases was higher than in the abovementioned studies. We previously reported that peripheral retinal nonperfusion correlates significantly with intravitreal ranibizumab injections in patients with BRVO and macular edema. ${ }^{33}$ Rehak et $\mathrm{al}^{34}$ evaluated 22 CRVO patients in a randomized clinical trial and suggested the selective laser photocoagulation of peripheral areas of nonperfusion may further improve the visual outcome and decrease the number of needed ranibizumab reinjections in CRVO patients.

This study has some limitations. There are only few published articles regarding the size of peripheral retinal nonperfusion using UWFA. We calculated the mean size of peripheral retinal nonperfusion for all enrolled patients, which was five disc areas, and then the patients were divided into two groups according to that cut-off point. However, the authors totally agree that this approach is not an established step due to lack of evidence. Moreover, there are several problems in the interpretation of fluorescein angiograms. The grading of the ultrawide-field angiograms is not uniform because there are eyelash artifacts that influence the entire amount of interpretable fundus, and the graders can also change the clarity of the images that may cause intergrader variability. Another drawback of our study design was that we performed UWFA for each patient at only one single time point. Thus, we were not able to demonstrate potential dynamic angiographic changes in nonperfusion areas over the follow-up time.

\section{Conclusion}

In conclusion, UWFA is a precise tool for the detection and quantification of retinal nonperfusion which correlated with the number of needed intravitreal ranibizumab injections in our patients with CRVO. Retinal nonperfusion could be considered as a prognostic factor for a more precise patient management.

\section{Disclosure}

The authors report no conflicts of interest in this work.

\section{References}

1. Klein R, Klein BE, Moss SE, Meuer SM. The epidemiology of retinal vein occlusion: the Beaver Dam Eye Study. Trans Am Ophthalmol Soc. 2000;98:133-141.

2. Mitchell P, Smith W, Chang A. Prevalence and associations of retinal vein occlusion in Australia. The Blue Mountains Eye Study. Arch Ophthalmol. 1996;114:1243-1247.

3. Hayreh SS. Prevalent misconceptions about acute retinal vascular occlusive disorders. Prog Retin Eye Res. 2005;24(4):493-519.
4. Green WR, Chan CC, Hutchins GM, Terry JM. Central retinal vein occlusion: a prospective histopathologic study of 29 eyes in 28 cases. Trans Am Ophthalmol Soc. 1981;79:371-422.

5. Ramchandran RS, Fekrat S, Stinnett SS, Jaffe GJ. Fluocinolone acetonide sustained drug delivery device for chronic central retinal vein occlusion: 12-month results. Am J Ophthalmol. 2008;146:285-291.

6. Heier JS, Campochiaro PA, Yau L, et al. Ranibizumab for macular edema due to retinal vein occlusions: long-term follow-up in the HORIZON trial. Ophthalmology. 2012;119:802-809.

7. Brown DM, Campochiaro PA, Singh RP, et al. Ranibizumab for macular edema following central retinal vein occlusion: six-month primary end point of a phase III study. Ophthalmology. 2010;117:1124-1133.

8. Holz FG, Roider J, Ogura Y, et al. VEGF Trap-Eye for macular oedema secondary to central retinal vein occlusion: 6-month results of the phase III GALILEO study. Br J Ophthalmol. 2013;97:278-284.

9. Tsui I, Kaines A, Havunjian MA, et al. Ischemic index and neovascularization in central retinal vein occlusion. Retina. 2011;31:105-110.

10. Boyd SR, Zachary I, Chakravarthy U, et al. Correlation of increased vascular endothelial growth factor with neovascularization and permeability in ischemic central vein occlusion. Arch Ophthalmol. 2002; 120:1644-1645.

11. Noma H, Funatsu H, Mimura T, Harino S, Hori S. Vitreous levels of interleukin-6 and vascular endothelial growth factor in macular edema with central retinal vein occlusion. Ophthalmology. 2009;116(1): 87-93.

12. Staurenghi G, Viola F, Mainster MA, Graham RD, Harrington PG. Scanning laser ophthalmoscopy and angiography with a wide-field contact lens system. Arch Ophthalmol. 2005;123:244-252.

13. Noyori KS, Chino K, Deguchi T. Wide field fluorescein angiography by use of contact lens. Retina. 1983;3:131-134.

14. Witmer MT, Parlitsis G, Patel S, Kiss S. Comparison of ultra-widefield fluorescein angiography with the Heidelberg Spectralis ${ }^{\circledR}$ noncontact ultra-widefield module versus the Optos ${ }^{\circledR}$ Optomap ${ }^{\circledR}$. Clin Ophthalmol. 2013;7:389-394.

15. Manivannan A, Plskova J, Farrow A, et al. Ultra-wide-field fluorescein angiography of the ocular fundus. Am J Ophthalmol. 2005;140(3): $525-527$.

16. Friberg TR, Gupta A, Yu J, et al. Ultrawide angle fluorescein angiographic imaging: a comparison to conventional digital acquisition systems. Ophthalmic Surg Lasers Imaging. 2008;39:304-311.

17. Witmer M, Kiss S. Wide-field imaging of the retina. Surv Ophthalmol. 2013;58(2):143-154.

18. Prasad PS, Oliver SC, Coffee RE, Hubschman JP, Schwartz SD. Ultra wide-field angiographic characteristics of branch retinal and hemicentral retinal vein occlusion. Ophthalmology. 2010;117(4):780-784.

19. Wessel MM, Aaker GD, Parlitsis G, Cho M, D'amico DJ, Kiss S. Ultra-wide-field angiography improves the detection and classification of diabetic retinopathy. Retina. 2012;32(4):785-791.

20. Wessel MM, Nair N, Aaker GD, Ehrlich JR, D'Amico DJ, Kiss S. Peripheral retinal ischaemia, as evaluated by ultra-widefield fluores $\neg$ cein angiography, is associated with diabetic macular oedema. Br J Ophthalmol. 2012;96(5):694-698.

21. Mudvari SS, Virasch VV, Singa RM, MacCumber MW. Ultra-widefield imaging for cytomegalovirus retinitis. Ophthalmic Surg Lasers Imaging. 2010;41(3):311-315.

22. Cho M, Kiss S. Detection and monitoring of sickle cell retinopathy using ultra wide-field color photography and fluorescein angiography. Retina. 2011;31(4):738-747.

23. Pe'er J, Sancho C, Cantu J, et al. Measurement of choroidal melanoma basal diameter by wide-angle digital fundus camera: a comparison with ultrasound measurement. Ophthalmologica. 2006;220(3):194-197.

24. Shields CL, Materin M, Shields JA. Panoramic imaging of the ocular fundus. Arch Ophthalmol. 2003;121(11):1603-1607.

25. Witmer MT, Cho M, Favarone G, Chan RV, D'Amico DJ, Kiss S. Ultrawide-field autofluorescence imaging in non-traumatic rhegmatogenous retinal detachment. Eye (Lond). 2012;26(9):1209-1216. 
26. Fung TH, Yusuf IH, Smith LM, Brett J, Weston L, Patel CK. Outpatient Ultra wide-field intravenous fundus fluorescein angiography in infants using the Optos P200MA scanning laser ophthalmoscope. Br J Ophthalmol. 2014;98:302-304.

27. Kaneko Y, Moriyama M, Hirahara S, Ogura Y, Ohno-Matsui K. Areas of nonperfusion in peripheral retina of eyes with pathologic myopia detected by ultra-widefield fluorescein angiography. Invest Ophthalmol Vis Sci. 2014;55(3):1432-1439.

28. Suñer IJ, Peden MC, Hammer ME, Grizzard WS, Traynom J, Cousins SW. RaScaL: a pilot study to assess the efficacy, durability, and safety of a single intervention with ranibizumab plus peripheral laser for diabetic macular edema associated with peripheral nonperfusion on ultrawide-field fluorescein angiography. Ophthalmologica. 2015; 233:89-95.

29. Kolar P. Risk factors for central and branch retinal vein occlusion: a meta-analysis of published clinical data. J Ophthalmol. 2014;2014: 724780 .
30. Zhou JQ, Xu L, Wang S, et al. The 10-year incidence and risk factors of retinal vein occlusion: the Beijing eye study. Ophthalmology. 2013; 120(4):803-808.

31. Spaide RF. Peripheral areas of nonperfusion in treated central retinal vein occlusion as imaged by wide-field fluorescein angiography. Retina. 2011; 31(5):829-837.

32. Singer M, Tan CS, Bell D, Sadda SR. Area of peripheral retinal nonperfusion and treatment response in branch and central retinal vein occlusion. Retina. 2014;34(9):1736-1742.

33. Abri Aghdam K, Reznicek L, Soltan Sanjari M, et al. Peripheral retinal non-perfusion and treatment response in branch retinal vein occlusion. Int J Ophthalmol. 2016;9(6):858-862.

34. Rehak M, Tilgner E, Franke A, Auscher FG, Brosteanu O, Wiedemann P. Early peripheral laser photocoagulation of nonperfused retina improves vision in patients with central retinal vein occlusion (Results of a proof of concept study). Graefes Arch Clin Exp Ophthalmol. 2014;252(5): $745-752$.
Clinical Ophthalmology

\section{Publish your work in this journal}

Clinical Ophthalmology is an international, peer-reviewed journal covering all subspecialties within ophthalmology. Key topics include: Optometry; Visual science; Pharmacology and drug therapy in eye diseases; Basic Sciences; Primary and Secondary eye care; Patient Safety and Quality of Care Improvements. This journal is indexed on

\section{Dovepress}

PubMed Central and CAS, and is the official journal of The Society of Clinical Ophthalmology (SCO). The manuscript management system is completely online and includes a very quick and fair peer-review system, which is all easy to use. Visit http://www.dovepress.com/ testimonials.php to read real quotes from published authors. 